\title{
PARAMETRIC SURFACES
}

\section{A. S. BESICOVITCH}

I want first to express my thanks to the Presidium of the American Mathematical Society for the invitation to deliver an address to the Society. I feel very much honored by this invitation and I appreciate it highly.

My address will be devoted to parametric surfaces, to that part of the subject which is connected with the definition of the area, with the expression of the area by an integral, and with the minimum area problem. It is an exceptional privilege to lecture on this subject to the American Mathematical Society, whose members have been responsible for such an impressive progress of the subject during the last two decades. I shall start with the definition of the area.

In 1914 Carathéodory defined the $m$-dimensional measure in the $n$-dimensional space, for integral values of $m .^{1} \mathrm{He}$ considered the one-dimensional measure as a generalization of the length and he proved that the length of a rectifiable curve coincides with its onedimensional measure.

In 1919, Hausdorff, ${ }^{2}$ developing Carathéodory's ideas, constructed a continuous scale of measures. After the work of Carathéodory and Hausdorff the obvious problem was to treat the area as the two-dimensional measure and to establish the well known integral formulae. But a considerable amount of work on establishing the integral formulae for the area had been done before that, with a variety of definitions of the area, due no doubt to the difficulty of the problem. Later the Lebesgue definition, somewhat modified by Frechet, of the area as the lower limit of areas of approximating polyhedra became the dominant one. A particularly valuable feature of the LebesgueFrechet (L.-F.) definition is that it provides the area with the property of the lower semi-continuity.

When applied to plane figures bounded by a Jordan curve the L.-F. definition gives in fact the value of the interior area of the figure, and thus in cases when the boundary is of positive $\mathrm{L}_{2}$-measure, the L.-F. area differs from the value of the closed area in the usual

An address delivered before the Annual Meeting of the Society at Columbus, Ohio, December 29, 1948, by invitation of the Committee to Select Hour Speakers for Summer and Annual Meetings; received by the editors April 14, 1949.

1 Über das lineare Mass von Punktmengen-eine Verallgemeinerung des Längenbegriffs, Nachr. Ges. Wiss. Göttingen (1914) pp. 404-426.

2 Dimension und ausseres Mass, Math. Ann. vol. 79 (1919) pp. 157-179. 
Lebesgue sense. This of course need not be considered in any sense a serious fault with the definition, and of course there is nothing astonishing then if the area of the projection measured as a closed area happens to be greater than the area of the projective figure measured as an open area. This is the so-called projected conflict, ${ }^{8}$ conflict only in name. Being entirely based on an obvious confusion of two measures, it could not have shaken any confidence in the L.-F. definition. As a matter of fact there is no conflict whatsoever, and the L.-F. area of the orthogonal projection of a parametric surface, which (the projection) is itself a parametric surface, is always less than or equal to the L.-F. area of the projected figure. This is obvious for polyhedral surfaces, and then it follows at once for the general case.

In 1913, Geöcze ${ }^{4}$ gave an example of a parametric surface that fills up a cube and has at the same time L.-F. area equal to zero. But the Geöcze parametric surface is no surface in any geometric sense and the notion of its area is entirely artificial. Whatever value might have been obtained for its area, it could not have been a test for the definition. Thus no fault was suspected in the L.-F. definition until recently, and a large amount of work was done on the basis of the L.-F. definition, culminating in the proof of the integral formula for parametric surfaces under the most general conditions, and in the solution of the Plateau problem. But the study of the area cannot be restricted to the very narrow class of the parametric surfaces, that is, to the class of continuous images of a disk. A notion of area is attached to a much wider class of surfaces, such as continuous images of other figures than a disk, many cases of discontinuous images and to surfaces given as point sets. With the experience of more than fifty years of modern theory of functions of a real variable, any attempt of extending the definition of the area to more and more general classes of surfaces would inevitably lead to treating the area as a measure, just as the length and the volume are treated.

A few years ago I set myself the problem of treating the area as the Hausdorff $\Lambda^{2}$-measure and of proving the identity of the L.-F. area of parametric surfaces and of their $\Lambda^{2}$-measure (excluding the boundaries). However I came to results very different from ones I was hoping for. In my paper, On the definition and the value of the area of a surface, ${ }^{5}$ I constructed a topological disk and a topological

${ }^{3}$ T. Rad6, On the problem of Plateau, Ergebnisse der Mathematik und ihre Grenzgebiete, vol. 2, 1933.

${ }^{4} \mathrm{Z}$. Geöcze, Sur l'exemple d'une surface dont l'aire est egale a zero et qui remplit un cube, Bull. Soc. Math. France vol. 41 (1913) pp. 29-31.

${ }^{5}$ Quart. J. Math. Oxford Ser. vol. 16 (1945) pp. 86-102. 
sphere whose surface, on the one hand, has L.-F. area as small as we please, and, on the other hand, has a positive Lebesgue three-dimensional measure. In order to avoid too complicated diagrams, I shall first construct a plane curve to which the required surface is very similar. Let $S_{0}=A$ be a unit square (Fig. 1). Divide $A$ into four equal squares and let $A_{1}, A_{2}, A_{3}, A_{4}$ be four smaller equal squares concentric with the above squares and of total area greater than $1 / 2$. Write $S_{1}=\sum_{1}^{4} A_{i_{1}}$, meaning by $S_{1}$ the set of interior and boundary points of the squares $A_{1}, \cdots, A_{4}$. Similarly a set $S_{2}$

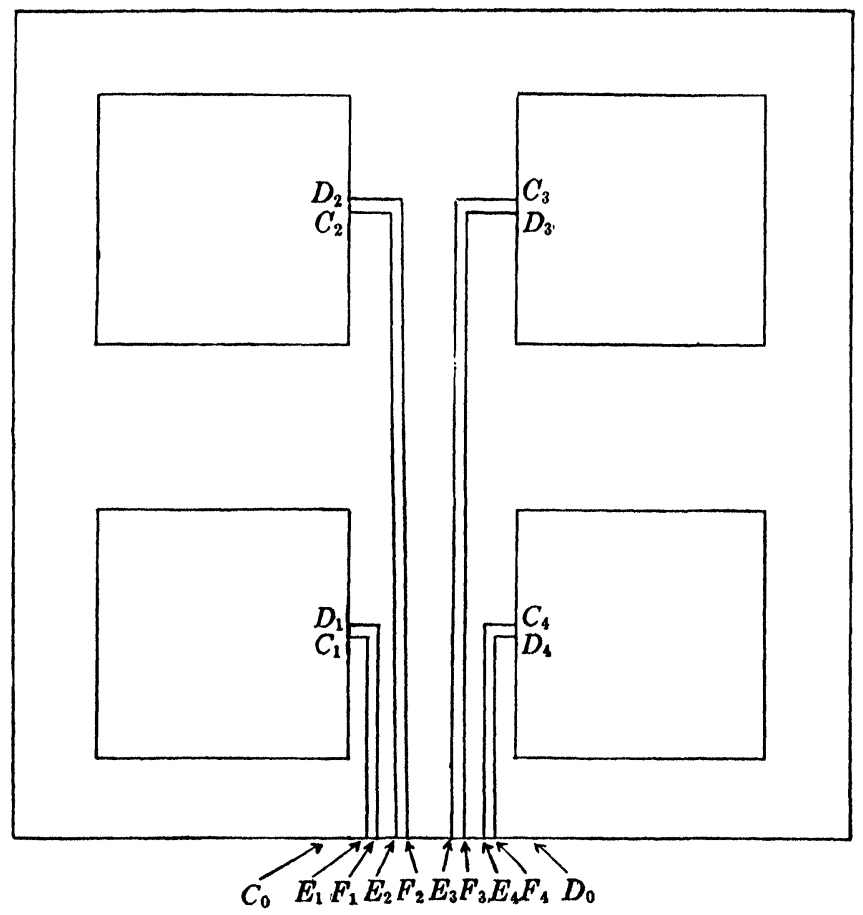

FIG. 1.

$=\sum_{i_{1}=1, \cdots, 4, i_{2}=1, \cdots, 4} A_{i_{1} i_{2}}$ of 16 equal squares of total area greater than $1 / 2$ and less than the total area of $S_{1}$ are constructed, four squares in each square of $S_{1}$, and so on. In this way a decreasing sequence $\left\{S_{n}\right\}$ is determined, where $S_{n}$ is a set of $4^{n}$ equal squares of total area greater than $1 / 2$. Writing $S=\lim S_{n}$, we have $m^{2} S \geqq 1 / 2\left(m^{2}\right.$ being the Lebesgue two-dimensional measure). Take now a segment $C_{0} D_{0}$ on a side of the square $A$ and segments $C_{1} D_{1}, \cdots, C_{4} D_{4}$ on sides of $A_{1}, \cdots, A_{4}$ and construct a polygonal line $\Pi_{1}=P_{0}$ $=C_{0} E_{1} C_{1} D_{1} F_{1} E_{2} C_{2} D_{2} F_{2} \cdots D_{4} F_{4} D_{0}$ of four branches. Similarly on 
each of the segments $C_{1} D_{1}, \cdots, C_{4} D_{4}$ construct polygonal lines $P_{1}, \cdots, P_{4}$, each of four branches. Replacing the sides $C_{1} D_{1}, \cdots$, $C_{4} D_{4}$ of $\Pi_{1}$ by the polygonal lines $P_{1}, \cdots, P_{4}$, we shall expand the line $\Pi_{1}$ to a line $\Pi_{2}$ of four branches of the first order and 16 branches of the second order. Similarly $\Pi_{2}$ is expanded to $\Pi_{3}, \Pi_{3}$ to $\Pi_{4}$, and so on. The sequence of polygonal lines $\left\{\Pi_{n}\right\}$ defines a limit curve $\Pi$, which is obviously a simple curve. The line $\Pi_{n}$ has a segment in common with every square of $S_{n}$, and consequently every point of $S_{n}$, and in particular every point of $S$ is within $2^{-n+1 / 2}$ from $\Pi_{n}$. Hence every point of $S$ is on $\Pi$. Thus $\Pi$ is a simple curve of infinite length and it contains the set $S$, whose two-dimensional measure is not less than $1 / 2$.

Now we carry out a similar construction in the three-dimensional space. Let $S_{0}=A$ be a unit-cube, and $S_{1}=\sum_{i=1}^{8} A_{i_{1}}$ a set of 8 equal cubes of total volume less than 1 and greater than $1 / 2$, symmetrically situated in $A$, by which we mean that the cubes $A_{i_{1}}, i_{1}=1, \cdots, 8$, are concentric with the eight cubes into which $A$ is divided by the three mid-planes. Let further $S_{2}=\sum_{i_{1}=1,2, \ldots, 8, i_{2}=1,2, \cdots, 8} A_{i_{1} i_{2}}$ be 64 equal cubes of total volume less than that of $S_{1}$ and greater than $1 / 2$, the cubes $A_{i_{1} 1}, \cdots, A_{i_{1} 8}$ being symmetrically situated in $A_{i_{1}}$, and so on. In this way a decreasing sequence of sets $S_{n}$ is determined. Writing $S=\lim S_{n}$, we have $m^{3} S \geqq 1 / 2\left(m^{3}\right.$ being the Lebesgue 3 -dimensional measure) since $m^{3} S_{n}>1 / 2$ for every $n$.

On the bottom side of $A$ take a small square $C_{0} D_{0}\left(C_{0}, D_{0}\right.$ are a pair of opposite vertices) and on $C_{0} D_{0}$ eight smaller disjoint squares $E_{1} F_{1}, \cdots, E_{8} F_{8}$, then take squares $C_{1} D_{1}, \cdots, C_{8} D_{8}$ on the bottom sides of the cubes $A_{1}, \cdots, A_{8}$ and join them to $E_{1} F_{1}, \cdots, E_{8} F_{8}$ respectively by rectangular tubes, that do not meet one another. The part $C_{0} D_{0}-\sum_{1}^{8} E_{i} F_{i}$ of the square $C_{0} D_{0}$, the eight tubes constructed above, and the squares $C_{1} D_{1}, \cdots, C_{8} D_{8}$ form a polyhedral surface $P_{0}$ of eight branches. Call it $\Pi_{1} . \Pi_{1}$ is a topological disk. Similarly polyhedral surfaces $P_{1}, \cdots, P_{8}$, of eight branches each, will be constructed on the squares $C_{1} D_{1}, \cdots, C_{8} D_{8}$ respectively. Replacing the squares $C_{1} D_{1}, \cdots, C_{8} D_{8}$ of $\Pi_{1}$ by the polyhedral surfaces, $P_{1}, \cdots, P_{8}$ respectively, we shall get a polyhedral surface $\Pi_{2}$ of eight branches of the first order and 64 branches of the second order. $\Pi_{2}$ is also a topological disk. Similarly $\Pi_{2}$ will be "expanded" to $\Pi_{3}, \Pi_{3}$ to $\Pi_{4}$, and so on. The sequence of surfaces $\Pi_{n}$ converges in the Frechet sense to a surface $\Pi$. $\Pi$ is a topological disk. Just as in the case of the plane construction $I I$ contains all the points of $S$, so that $m^{3} \Pi \geqq 1 / 2$. Now comes a fundamental difference between the plane figure and the three-dimensional one. While in the case of the plane figure the length of the polygonal line $\Pi_{n}$ tends to infinity, in the 
case of the three-dimensional figure the areas of the polyhedral surfaces $\Pi_{1}, \Pi_{2}, \cdots$ may be as small as we please, so that given $\epsilon>0$ we may assume

$$
\lim \Lambda^{2} \Pi_{n}<\epsilon
$$

and hence L.-F. area of $I$ less than $\epsilon$.

(i) Thus we have constructed a topological disk, whose area, in the L.-F. definition, is less than $\epsilon$, and whose three-dimensional Lebesgue measure is not less than $1 / 2$.

(ii) If we seal the surface $\Pi$ by a surface of small area subtending the boundary of the square $C_{0} D_{0}$ and lying below it, we shall get a topological sphere $\Pi^{\prime}$. The volume of $\Pi^{\prime}$ (that is, $m^{3}$-measure) is greater than $1 / 2$ and the area of the surface of $\Pi^{\prime}$ may be as small as we please. The solid bounded by the surface $\Pi^{\prime}$ has different values for the upper volume and for the lower volume. Therefore at preLebesgue time it would have been considered that the volume was not defined for this solid, and the surface $\Pi^{\prime}$ would have had no bearing on the classical isoperimetric problem of finding the solid of maximum volume, bounded by a surface of a given area. However, since the development of methods of measure the notions of the upper and lower volumes have lost their relevance and the volume is defined as the $m^{3}$-measure. Therefore the result presented by $\Pi^{\prime}$ is in conflict with the existing solution of the above problem. The result (i) is in conflict with all geometrical ideas of the area:

(a) It shows that the L.-F. area cannot be fitted into any scale of measures.

(b) It has no property of additivity, even in the most clear cases. A construction similar to one employed above leads to a parametric surface that includes all points of a unit-square, and whose area is as small as we please.

(c) It has not been, and it cannot be, extended to surfaces given as point-sets. For these reasons, clinging to the L.-F. definition of area would imply treating it as a functional defined in an artificially restricted domain and completely detached from the general study of the area.

The general study of area can be brought to the standards of perfection of the modern theory of functions of a real variable only by treating the area as a measure. I define the area as the Hausdorff two-dimensional measure (with a corrective for the case of multiple points), and I solve anew the fundamental problems on area: that of integral expression of the area and the Plateau problem. The right principle brings a generous reward: tremendous topological complica- 
tions for establishing the integral formulae are avoided completely, the integral formulae are valid not only for the whole surface, but also for sets of points on the surface; in the case of the Plateau problem all restrictions on the contour are dropped. In the solution of the Plateau problem I was helped by having discovered conditions of compactness and of semi-continuity of the area in the class of parametric surfaces.

Before going further into the problems of my lecture, we have to introduce strict definitions concerning parametric surfaces.

We shall consider continuous functions $P=\Phi(M)$ whose domain of definition is a disk $H$ and whose values are points in the threedimensional space. For any point $P$, the set $\Phi^{-1}(P)$ is closed. Any component of $\Phi^{-1}(P)$ is called a $\Phi$-element of $H$. Any $\Phi$-element is either a point or a saturated continuum in $\Phi^{-1}(P)$. Thus the equation $P=\Phi(M)$ defines a representation $H=\sum Q$ of $H$ as the sum of $\Phi$-elements. Any pair of $\Phi$-elements are either coincident or have no points in common. We form pairs $(P, Q)$ where $Q$ are $\Phi$-elements, and, for every $Q, P$ is the value of $\Phi(M)$ on $Q$. The set $\Pi=\sum(P, Q)$ of all such pairs is called the parametric surface defined by the equation $P=\Phi(M)$ (or by the function $\Phi(M)$ ) and the pairs $(P, Q)$ the points of the parametric surface.

If a set $E \subset H$ is given, the symbol $\Phi(E)$ will be used in two different senses: it is the set of values of $\Phi(M)$ at all points of $E$, and also it is the set $\sum(P, Q)$ of points of $\Pi$ for all $\Phi$-elements $Q$ such that $Q \cdot E \neq 0$. In either case it is "the image of $E$."

Given two points $X_{i}=\left(P_{i}, Q_{i}\right)(i=1,2)$, let $c$ be any continuum in $H$ joining $Q_{1}$ and $Q_{2}$. The lower bound of the diameter of $\Phi(c)$ for all possible $c$ is called the $\Pi$-distance between $X_{1}$ and $X_{2}$, and is denoted by $D_{\mathrm{I}}\left(X_{1}, X_{2}\right)$. If $Q_{1} \neq Q_{2}, D_{\mathrm{II}}\left(X_{1}, X_{2}\right)>0$ whether $P_{1}$ is different from $P_{2}$ or not.

If there are exactly $k$ points of $\Pi,\left(P, Q_{1}\right), \cdots,\left(P, Q_{k}\right)$, with the same first element, then $P$ is said to be of multiplicity $k$. The $k$ points are distinct and are at a positive II-distance from one another.

Given two parametric surfaces $\Pi_{1}$ and $\Pi_{2}$ defined by the functions $\Phi_{1}(M)$ and $\Phi_{2}(M)$, let $\Psi(M)$ be any homeomorphism on $H$. Let $D(\Psi)$ be the maximum of the Euclidean distance between the points $\Phi_{1}(M)$ and $\Phi_{2}\{\Psi(M)\}$ as $M$ varies in $H$. The lower bound of $D(\Psi)$ for all $\Psi(M)$ is called the $F$-distance (Frechet distance) between $\Pi_{1}$ and $\Pi_{2}$ and is denoted $D_{F}\left(\Pi_{1}, \Pi_{2}\right)$. If $D_{F}\left(\Pi_{1}, \Pi_{2}\right)=0$, we say that $\Pi_{1}$ and $\Pi_{2}$ are identical parametric surfaces. Thus the surfaces defined by $\Phi(M)$ and by $\Phi\{\Psi(M)\}$ are identical. $F$-distance can be defined in a different way, in terms of $\Phi$-elements, but we shall not dwell on that. Once the 
distance between parametric surfaces is defined, convergent sequences of parametric surfaces and limit parametric surfaces are defined too. We have a simple theorem:

THEOREM. A necessary and sufficient condition for a sequence $\left\{\Pi_{n}\right\}$ to be convergent is that it should be representable by a uniformly convergent sequence $\left\{\Phi_{n}(U)\right\}$ of functions.

A set $E(\delta, \Pi)$ of points of $\Pi$ such that no pairs of them are within $\delta$ from each other and that any point of $\Pi$ is within $\delta$ of at least one point of $E$ is called a $\delta$-set of $\Pi$. $A \delta$-set is always finite.

We shall be considering aggregates of parametric surfaces in a bounded part of the space. We shall assume them bounded. Given an aggregate $A$, if there exists a finite function $N(\delta)$ of a positive variable $\delta$ such that, for any $\Pi \in A, \mathcal{N} E(\delta, \Pi)<N(\delta)$ ( $\mathcal{N}$ stands for the number of points of $E$ ) we say that $A$ is uniformly bounded with respect to neighborhoods.

A plane domain $G$ whose boundary consists of a finite number of disjoint Jordan curves is called a regular domain. A representation $H=\sum_{1}^{n} \bar{G}_{i}$ where $G_{1}, \cdots, G_{n}$ are disjoint regular domains is called a regular dissection of $H$. Given a regular domain $G$ in $H, \Phi(G)$ will be called a regular domain in $\Pi$. Similarly, to a regular dissection $H=\sum_{1}^{n} \bar{G}_{i}$ corresponds a regular dissection $\Pi=\sum \Phi\left(\bar{G}_{i}\right)$ of $\Pi$.

We can now state the main results concerning our problems. We shall start with compactness. An aggregate of parametric surfaces is said to be compact if any sequence of parametric surfaces of the aggregate contains a convergent subsequence. A complete solution of the compactness problem is given by the following theorem.

Theorem. $A$ necessary and sufficient condition for an aggregate $A$ to be compact is that it should be uniformly bounded with respect to neighborhoods.

The proof of the theorem depends partly on the following problem.

Problem. To divide $\Pi$ into regular domains of small diameters and to estimate the number of domains.

This can be done in terms of the number $n$ of points of the set $\mathcal{E}(\delta, \Pi)$. The problem is more difficult than it looks. It seems that if we take $\delta$-neighborhoods of points of $E(\delta, \Pi)$, they would give a solution. But these neighborhoods are overlapping, and if we try to reduce them for eliminating the overlapping, then this, in general, leads to dividing some of the neighborhoods into many disjoint ones. The actual answer to the problem is given by the following lemma. 
LEMMA 1. II can be represented as the sum of not more than $n$ regular domains each of diameter less than $50 \delta$.

This lemma becomes effective when coupled with the following lemma.

LEMma 2. The number of topologically different dissections of $H$ into $m$ regular domains is finite for every $m$.

The next problem is that of lower semi-continuity of area. Let $U$ be the set of the first elements of the points of the parametric surface $\Pi$ defined by the equation $P=\Phi(M)$. Write $U=\sum_{1}^{\infty} U_{k}$, where $U_{k}$ is the set of points of $\Pi$ of multiplicity $k$. Measure of $\Pi$ is defined by the sum

$$
\Lambda^{2} \Pi=\sum_{k=1}^{\infty} k \Lambda^{2} U_{k},
$$

where the term corresponding to $k=\infty$ is 0 if $\Lambda^{2} U_{k}=0$, and $\infty$ if $\Lambda^{2} U_{k}>0$. Similarly $\Lambda^{2} \Phi(E)$ is defined for any $E \subset H$. The measure defined in this way obviously has the property of additivity.

A parametric surface is called smooth if any part of $\Pi$ (if any) whose boundary lies in a plane lies itself in a plane.

The lower semi-continuity theorem is established in the following form.

If $\Pi=\lim \Pi_{n}$, then $\Lambda^{2} \Pi^{0} \leqq \lim$ inf $\Lambda^{2} \Pi_{n}^{0}\left(\Pi^{0}, \Pi_{n}^{0}\right.$ are the sets of interior points of $\mathrm{\Pi}, \mathrm{I}_{n}$ ) in two cases:

(i) when II is a smooth surface;

(ii) when $\Pi$ has a tangential plane at almost all points in the sense of $\Lambda^{2}$-measure.

Thus it is established only for interior areas and it is not true for the closed areas.

Having properties of compactness and of lower semi-continuity, we come in an obvious way to a proof of existence of a surface of minimum area subtending an arbitrary parametric contour $\Gamma$.

Let $a=1$.bd $\Lambda^{2} \Pi$, where $\Pi$ is any surface subtending $\Gamma$, and let $\left\{\Pi_{n}\right\}$ be a sequence of surfaces subtending $\Gamma$ and such that $\Lambda^{2} \Pi_{n} \rightarrow a$. By smoothing operations, which may only reduce the value of the area, every $\Pi_{n}$ may be replaced by a smooth surface of area not greater than $\Lambda^{2} \Pi_{n}$. Thus we may simply assume all the surfaces $\Pi_{n}$ to be smooth. In this case the aggregate $\left\{\Pi_{n}\right\}$ is easily seen to be uniformly bounded with respect to neighborhoods. Consequently, the compactness theorem is applicable. Thus we can assume the sequence $\left\{\Pi_{n}\right\}$ convergent. Let $\Pi=\lim \Pi_{n} ; \Pi$ is obviously smooth and 
thus the lower semi-continuity theorem is applicable:

$$
\Lambda^{2} \Pi^{0} \leqq \lim \inf \Lambda^{2} \Pi_{n}^{0} .
$$

We have to establish a similar relation for the boundaries, which, one may think, is obvious as the boundary is the same. This is true in the case when all the points of the boundary are simple. If the boundary has multiple points, then their multiplicity may depend on the surface subtending them and thus measure of the boundary may vary from surface to surface. Still the required relation can be easily established and the proof of the formula $\Lambda^{2} \Pi=a$ completed.

The second part of the Plateau problem, that of showing that II is a harmonic surface, follows in the usual way since according to Cesari for a smooth surface $\Pi, \Lambda^{2} \Pi$ coincides with the L.-F. area of $\Pi$.

Now only a few words about the last problem, that of expressing the value of the area in the form of an integral.

In the case of a non-parametric surface $z=f(x, y)$, the formula is established under the Tonelli conditions.

In the case of a parametric surface $P=\Phi(M)$, the integral formula holds if $\Phi(M)$ is approximately differentiable at almost all points of $H$ and if it is absolutely continuous in the sense that for any $E \subset H$ of $m^{2}$-measure zero, $\Lambda^{2} \Phi(E)=0$.

University of Pennsylvania 